\title{
A taxonomic analysis of miscellaneous fungi collected from Burma
}

\author{
Thaung $M^{1,2}$ \\ ${ }^{1} 5482$ Los Robles, La Verne, CA 91750-1643, USA. mmthaung@verizon.net \\ ${ }^{2}$ University and Jepson Herbaria/Botany, 1001 Valley Life Sciences Building \# 2465, University of California, \\ Berkeley, CA 94720-2465
}

Thaung MM 2015 - A taxonomic analysis of miscellaneous fungi collected from Burma. Mycosphere 6(1), 8-12, Doi 10.5943/mycosphere/6/1/2

\begin{abstract}
Records of fungi from Burma are updated to align with their current taxonomic nomenclature and classification. The update has generated a classified list of 58 contemporary species assigned to 27 families in 14 orders and 7 classes. This analysis aims at fungal taxa reported in world literature on Burma with a view to annotating and adding them to the local inventory for further enrichment and refinement.
\end{abstract}

Key words - aloewood - amber - biodiversity - Myanmar - systematics - truffle

\section{Introduction}

Fungi have been living in Burma in freedom since some 100 million years ago when a few of them had the misfortune of being entombed alive in Early Cretaceous Burmese amber (Poinar \& Buckley 2007). Their ancestors watched in silence as the dinosaurs roared, roamed, and ruled the earth. While some still remain entrapped in amber outside the plant (Poinar et al. 2014), others entered the wood and remained embedded to help generate rich fragrance for the world (Kurz 1877). At the other end of the spectrum, there lies hidden close to the roots, as little eggs or balls, a local truffle (Kaing-U or Kaing-Oo) in association with tall elephant grass (Pennistum purpureum Roem. \& Schult. or Saccharum arundinaceum Retz. = Kaing) in the tidal forests or in the Irrawaddy Delta (Troup 1913, Rodger 1936, Thaung 2007). This study also presents a supplement to an earlier work on macromycetes of Burma (Thaung 2007). The objective was to contribute to building capacity for contemporary mycodiversity information of the territory.

\section{Materials \& Methods}

The main target of this investigation was the 19th Century Kurz and Mason collections of Burman fungi initially identified by Kurz and subsequently by Currey (Currey 1875, Mason \& Theobald 1883). Their specimens are hardly accessible or traceable for traditional verification. However, they are long overdue for a systematic review and proper disposition following modern databases (www.indexfungorum.org/ and www.mycobank.org/). Therefore, these fungi are now reclassified and resubmitted below together with their original data to provide a contemporary and comparative taxonomic information.

\section{Results}

The records were reviewed and are enumerated below in alphabetical and hierarchical order. 


\section{Agaricomycetes, Basidiomycota}

Agaricaceae, Agaricales

1. Agaricus campestris L. 1753. On ground, Moulmein.

2. Cyathia intermedia (Mont.) V.S. White 1902 (= Cyathus intermedius (Mont.) Tul. \& C. Tul.1844) On ground, Moulmein.

3. Lycoperdon brasiliense Fr. 1829. Nakawa, Ton-kye-gat.

4. Lycoperdon pusillum Fr. (legitimate, year not available). Lower Pegu.

Marasmiaceae

1. Marasmius parishii Cooke (legitimate, year not available). On herbaceous plants and petioles of palm leaf, Moulmein.

Psathyrellaceae

1. Psathyrella candolleana (Fr.) Maire 1937 (=Agaricus candolleanus Fr. $1818=$ A. appendiculatus Bull. 1789, recorded inaccurately as A. (Hypholoma) appendiculatus Fr.). Moulmein.

Tricholomataceae

1. Pseudoclitocybe cyathiformis (Bull.) Singer 1956 (=Agaricus cinerascens Batsch 1786). Pegu Yoma.

Auriculariaceae, Auriculariales

1. Exidia auricula-canis (G. Mey.) Fr. 1822 (recorded as Hirneola auricula-canis Fr.). Northern Yoma, Pegu.

Incertae sedis

1. Guepinia ramosa Curr. 1876. Arakan State.

Paxillaceae, Boletales

1. Paxillus involutus (Batsch) Fr. 1838 (= Agaricus contiguus Bull. 1785 for Agaricus continuus Berk. 1847, illegitimate name). Moulmein.

Hymenochaetaceae, Hymenochaetales

1. Hymenochaete adusta (Lév.) Har. \& Pat. 1903 (= Stereum adustum Lév. 1844). Southern Yoma, Pegu.

2. Inonotus splitgerberi (Mont.) Ryvarden 1972 (= Polyporus splitgerberi Mont. 1841). Nattaung, Toungoo.

3. Polystictus persoonii Fr. Moulmein, Nakawa, Pegu, Ton-kye-gat.

Phallaceae, Phallales

1. Phallus daemonum Rumph. ex Fr. 1823. Moulmein, Pegu Yoma, Evergreen Forest

Fomitopsidaceae, Polyporales

1. Fomitella rhodophaea (Lév.) T. Hatt. 2005 (= Polyporus cinereofuscus Curr. 1876). Nakawa, Pegu, Ton-kye-gat.

2. Fomitopsis rubida (Berk.) A. Roy \& A.B. De [as 'rubidus'] 1996 (= Polyporus rubidus Berk. 1847). Pegu.

3. Laetiporus sulphureus (Bull.) Murrill 1920 (= Polyporus rubricus Berk. 1851). Pegu.

Meruliaceae

1. Podoscypha elegans (G. Mey.) Pat. 1900 (= Stereum elegans (G. Mey.) Fr. 1838). Lower Pegu. 
Polyporaceae

1. Lentinus badius (Berk.) Berk. 1847 (= Lentinus inquinans Berk. 1854). Kyi-myin-dine, Rangoon.

2. Lentinus coadunatus Hook. f. 1851. Pegu Yoma Range.

3. Lentinus exilis Klotzsch 1836. Nakawa, Pegu, Ton-kye-gat.

4. Lentinus irregularis Curr. 1876. Karen Area, Pegu Division, Pine Forests.

5. Lentinus kurzianus Curr. 1876. Pegu Yoma Range.

6. Lopharia papyrina (Mont.) Boidin 1959 (= Stereum papyrinum Mont. 1842). Pegu, Timeokee.

7. Microporus xanthopus (Fr.) Kuntze 1898 (= Polyporus xanthopus Fr. 1818, Polyporus florideus Berk. 1854). Bookee, Karen Hills, Moulmein, Nakawa, Pegu Yoma, Sittang Valley, Ton-kye-gat.

8. Panus neostrigosus Drechsler-Santos \& Wartchow 2012 (= Lentinus capronatus Fr. 1838), Myodwyn, Pegu.

9. Polyporus amboinensis Fr. 1821. Pegu, Karen Area.

10. Polyporus applanatus (Pers.) Wallr. 1833 (not Fr. which does not exist). Evergreen Forest, Pegu, Ton-kye-ghat, Toungoo.

11. Polyporus crassipes Curr. 1876. Pegu Yoma.

12. Pseudofavolus polygrammus (Mont.) G. Cunn. 1965 (= Hexagonia polygramma (Mont.) Fr. 1838). Pellowa, Ton-kye-gat.

13. Pycnoporus sanguineus (L.) Murrill. 1904 (= Polyporus sanguineus (L.) Fr. 1821). Moulmein.

14. Trametes palisotii (Fr.) Imazeki 1952 (= Lenzites palisotii (Fr.) Fr. 1838). Pegu, Seven-Pagoda Village (Toungoo), Ton-kye-gat.

15. Trametes umbrina Fr. 1845 (= Trametes umbrinus Curr. 1874, illegitimate name). Evergreen Forests, Nakawa, Pegu, Ton-kye-gat.

16. Trametes villosa (Sw.) Kreisel 1971 (= Polyporus pinsitus Fr. 1828). Nat-Taung, Toungoo.

Bondarzewiaceae, Russulales

1. Amylosporus campbellii (Berk.) Ryvarden 1977 (= Polyporus campbellii Berk. 1854, P. anthelminticus Berk. $1866=$ bamboo agaric $($ Wa-hmo), worm mushroom (Than-hmo), on the culms of bamboo near the ground. Tin-wa (= Cephalostachyum pergracile Munro 1868) or Kyatthaungwa (= Bambusa polymorpha Munro 1868) in Tharawaddy and Pegu Forests (Brandis 1903).

Lachnocladiaceae

1 Dichostereum granulosum (Pers.) Boidin \& Lanq. 1977 (= Grandinia granulosa (Pers.) Fr. 1838). Pegu, Pellowa, Ton-kye-gat.

Peniophoraceae

1. Peniophora violaceolivida (Sommerf.) Massee 1890 (= Corticium violaceolividum (Sommerf.)

Fr. 1838). Pegu Yoma.

Stereaceae

1. Stereum cyathiforme (Fr.) Fr. 1838 (recorded as Stereum cyathiforme Curr. 1876, illegitimate name). Bookee Ridges, Karen Hills, Pegu.

2. Xylobolus subpileatus (Berk. \& M.A. Curtis) Boidin 1958 (= Stereum scytale Berk.1854). Bookee Ridges, Karen Hills, Pegu.

\section{Fossil Agaricomycetes, Basidiomycota}

Incertae sedis, Agaricales

1. Palaeoagaricites antiquus Poinar \& R. Buckley [as 'Palaeoagaracites'] 2007 (Holotype No. Buckley AB-368, MycoBank \# MB510456 and MB510465) in Early Cretaceous Burmite. Hukawng Valley, SW of Maingkhwan, Kachin State. Oldest agaric in the world.

2. Mycetophagites atrebora Poinar \& R. Buckley 2007. As mycoparasite on pileus of host fungus, Palaeoagaricites antiquus Poinar \& R. Buckley 2007, in amber from Early Cretaceous Burma. 
3. Entropezites patricii Poinar \& R. Buckley 2007. As hypermycoparasite inside hyphae of host mycoparasite, Mycetophagites atrebora Poinar \& R. Buckley 2007, in Burmite.

Sclerodermataceae, Boletales

1. Palaeogaster micromorpha Poinar, Alfredo \& Baseia 2014 (MycoBank \# MB 801127). In earlymid Cretaceous amber. Collected in 1999 by an unknown amber miner (s.n.) from Amber mine in the Hukawng Valley, SW of Maingkhwan, Kachin State.

\section{Pezizomycetes, Ascomycota}

Sarcoscyphaceae, Pezizales

1. Cookeina tricholoma (Mont.) Kuntze 1891 per Lockwood (2013).

\section{Tuberaceae}

1. Kaing-U or Kaing-Oo (unidentified truffle). Around the roots of elephant grass in rainy season. Delta Area.

2. Tinyu/Tinshu-U (unidentified truffle). Around the roots of pine trees (Pinus khasya Royle). Shan State and Chin State.

\section{Sodariomycetes, Ascomycota}

Togniniaceae, Diaporthales

1. Phaeoacremonium parasiticum (Ajello, Georg \& C.J.K. Wang) W. Gams, Crous \& M.J. Wingf. 1996 (Anamorphic Togninia). Presumably inside the wood of Aquilaria agallocha Roxb. (agarwood, aloeswood, Ah-kyaw, eagle-wood) plants, inducing fragrance or incense production. Martaban hills east of Toungoo and Tenessarem forests.

Hypocreaceae, Hypocreales

1. Hypocrea variabilis Curr. 1876. On living leaves of bamboo, Pegu Yoma.

Diatrypaceae, Xylariales

1. Diatrype phaselina (Mont.) Rappaz 1987 (= Sphaeria phaselina Mont. 1855). Nakawa, Ton-kyegat.

2. Diatrype rugosa Curr. 1876. On hardwood, Yoma, Pegu.

Xylariaceae

1. Annulohypoxylon annulatum (Schwein.) Y.M. Ju, J.D. Rogers \& H.M. Hsieh 2005 (= Hypoxylon marginatum var. marginatum (Schwein.) Berk. 1860). Pegu.

2. Hypoxylon sublimbatum (Durieu \& Mont) P.M.D. Martin 1967 (= Sphaeria sublimbata Durieu \& Mont. 1848). Kambala Taung.

3. Poronia pileiformis (Berk.) Fr. 1851. Moulmein.

4. Xylaria flagelliformis Curr. 1876. On earth, Pegu, Sittaung Valley, Yoma Range.

5. Xylaria guianensis (Mont.) Fr. 1851. Evergreen Forest, Pegu, Ton-kye-gat.

\section{Incertae sedis, Ascomycota}

1. Fumago salicina Tul. \& C. Tul. 1863. Pegu Yoma, Wa Choung.

\section{Chytridiomycetes, Chytridiomycota}

Rhizophlyctidaceae, Rhizophlyctidales

1. Rhizophlyctis reynoldsii Dogma 1974. On chitin bait in leaf litter, Rangoon.

2. Rhizophlyctis variabilis Karling 1968 (= Rhizophlyctis variabilis var. burmaensis Dogma 1974).

On chitin bait in soil, Rangoon.

\section{Myxomycetes, Myxomycota}


Arcyriaceae, Trichiales

1. Arcyria umbrina Schumach. 1803 (recorded as Arcyna umbrina Fr. which does not exist). Evergreen Forests, Pegu, Pellowa, Ton-kye-gat.

\section{Discussion}

The Kurz and Mason collections were obtained largely from the Pegu Yoma region with Toungoo in the north and Rangoon and Moulmein in the south. This area was then very sparsely populated, heavily wooded, and almost pristine and untouched by human activities. The fleshy fungi abound there due to heavy monsoon rains for a greater part of the year. The forays yielded several new species including Diatrype rugosa, Guepinia ramosa, Hypocrea variabilis, Lentinus irregularis, L. kurzianus, Marasmius parashii, Nectria eugeniae, Polyporus cinereofuscus, P. crassipes, $P$. peguanusand Xylaria flagelliformis.

Moreover, the oldest agaric in the world resides within Early Cretaceous Burmite together with its mycoparasite and the hypermycoparasite (Poinar \& Buckley 2007). A bolete also remains confined inside of another amber from early-mid Cretaceous Burma (Poinar et al. 2014). Based on its unique location, association, infomercial data, and distinctive properties, agarwood fungus, Phaeoacremonium parasiticum, is suspected in the native wood of Aquilaria agallocha Roxb. (Ah$\begin{array}{llll}\text { kyaw } & = & \text { vernacular name) } & \text { (Kurz }\end{array}$ http://www.paradiseperfumes.com/agarwood_from_fungus_to_fragrance). It was thus presumed present without further ado. Three truffles join this medley, Tuber indicum Cooke \& Massee 1892 and two others known for over a century by local names only - Kaing-U/Kaing-Oo and Tinyu/Tinshu-U (Troup 1913, Thaung 2007). A bamboo fungus (Brandis 1903), a myxomycete and two chytrids (Dogma 1974) complete this snapshot of the day for miscellaneous fungi of Burma. And the mycoscape will be more interesting when the identities of the two truffles become available.

This paper provides a glimpse of Burma's rich tapestry of fossil, fragrance and food fungi for mycodiversity information.

\section{References}

Brandis D. 1903 - Notes on Gelsemium elegans and the bambo fungus of Burma. The Indian Forester 29, 404-406.

Currey F. 1875 - On a collection of fungi made by Mr. Sulpiz Kurz, Curator of the Botanic

Gardens, Calcutta. Transactions of Linnean Society, London, II Series, Botany 1, 119-131.

Dogma IJ. 1974 - Developmental and taxonomic studies on rhizophlyctoid fungi, Chytridiales III. Chitinophilic Rhizophlyctis with resting spores of sexual origin. Nova Hedwigia 25, 51-89.

Kurz S. 1877 - Forest Flora of British Burma. Vol. II. Office of the Superintendent of Government Printing, Calcutta.

Lockwood $\quad$ TF. $2013 \quad-\quad$ Cookeina tricholoma-Burma. http://www.fungiphoto.com/CTLG/pages/0851-07.html (accessed 8 October 2014).

Mason F, Theobald W. 1883 - Burma, its People and Productions. Vol. II. Stephen Austin \& Sons, Hertford, UK.

Poinar GO Jr, Buckley R. 2007 - Evidence of mycoparasitism and hypermycoparasitism in Early Cretaceous amber. Mycological Research 111, 503-506.

Poinar GO Jr, Alfredo DS, Baseia 1G. 2014 - A gasteroid fungus, Palaeogaster micromorpha gen. \& sp. nov. (Boletales) in cretaceous Myanmar amber. Journal of the Botanical Research Institute of Texas 8, 139-143.

Rodger A. 1936 - A hand-book of the forest products of Burma. Superintendent, Government Printing and Stationery, Burma. 166 pp.

Thaung MM. 2007 - A preliminary survey of macromycetes in Burma. Australasian Mycologist 26, $16-36$.

Troup RS. 1913 - Indian Forest Utilization, $2^{\text {nd }}$ edition (revised). Superintendent of Government Printing, Calcutta. 